\title{
$K$-THEORY AND HOMOTOPIES OF 2-COCYCLES ON GROUP BUNDLES
}

\author{
ELIZABETH GILLASPY
}

\begin{abstract}
This paper continues the author's program of investigating the question of when a homotopy of 2cocycles $\Omega=\left\{\omega_{t}\right\}_{t \in[0,1]}$ on a locally compact Hausdorff groupoid $\mathcal{G}$ induces an isomorphism of the $K$-theory groups of the twisted groupoid $C^{*}$-algebras:

$$
K_{*}\left(C^{*}\left(\mathcal{G}, \omega_{0}\right)\right) \cong K_{*}\left(C^{*}\left(\mathcal{G}, \omega_{1}\right)\right)
$$

Building on our earlier work in $[4,5]$, we show that, if $\pi: \mathcal{G} \rightarrow M$ is a locally trivial bundle of amenable groups over a locally compact Hausdorff space $M$, a homotopy $\Omega=$ $\left\{\omega_{t}\right\}_{t \in[0,1]}$ of 2-cocycles on $\mathcal{G}$ gives rise to an isomorphism:

$$
K_{*}\left(C^{*}\left(\mathcal{G}, \omega_{0}\right)\right) \cong K_{*}\left(C^{*}\left(\mathcal{G}, \omega_{1}\right)\right)
$$
\end{abstract}

1. Introduction. Let $\pi: V \rightarrow M$ be a real or complex vector bundle over a manifold $M$. A bilinear 2-form $\sigma: V^{(2)} \rightarrow \mathbb{R}$ induces a homotopy of 2-cocycles $\left\{\omega_{t}\right\}_{t \in[0,1]}$ on $V$. If $\pi(v)=\pi(w)$, define

$$
\omega_{t}(v, w):=e^{2 \pi i t \sigma(v, w)} .
$$

Plymen [11, Theorem 1] proved that, when $V$ is an even-dimensional real vector bundle and $\sigma$ is a symplectic 2 -form on $V$, the twisted $C^{*}$-algebra of the vector bundle $C^{*}\left(V, \omega_{1}\right)$ is a continuous-trace $C^{*}$ algebra over $M$, with trivial Dixmier-Douady class, and hence is Morita equivalent to $C_{0}(M)$.

Furthermore, applying a fiberwise Fourier transform to $V$, one sees immediately that

$$
C^{*}\left(V, \omega_{0}\right)=C^{*}(V) \cong C_{0}\left(V^{*}\right)
$$

2010 AMS Mathematics subject classification. Primary 46L05, 46L80.

Keywords and phrases. Group bundle, 2-cocycle, twisted groupoid $C^{*}$-algebra, $K$-theory.

Received by the editors on August 5, 2014, and in revised form on October 23, 2014. 
where $V^{*}$ is the dual bundle to $V$. Since $V$ is even-dimensional, the Thom isomorphism in $K$-theory tells us that

$$
K_{*}\left(C_{0}\left(V^{*}\right)\right) \cong K_{*}\left(C_{0}(M)\right) ;
$$

consequently, Plymen's result implies that the homotopy of 2-cocycles $\left\{\omega_{t}\right\}_{t \in[0,1]}$ associated to a symplectic form $\sigma$ induces an isomorphism

$$
K_{*}\left(C^{*}\left(V, \omega_{0}\right)\right) \cong K_{*}\left(C^{*}\left(V, \omega_{1}\right)\right) .
$$

In this article, we present a substantial generalization of this result:

Corollary 3.4. Let $\left\{\omega_{t}\right\}_{t \in[0,1]}$ be a homotopy of 2-cocycles on a second countable, locally trivial, locally compact Hausdorff group bundle $\pi: \mathcal{G} \rightarrow M$, such that the fiber group $G=\pi^{-1}(m)$ is amenable. Then the homotopy induces an isomorphism

$$
K_{*}\left(C^{*}\left(\mathcal{G}, \omega_{0}\right)\right) \cong K_{*}\left(C^{*}\left(\mathcal{G}, \omega_{1}\right)\right)
$$

of the $K$-theory groups of the twisted $C^{*}$-algebras.

While the motivation (and main applications) of this result arise from considering vector bundles over manifolds, the proofs are no simpler in this special case. Consequently, we present the results here in their full generality.

Group bundles are examples of groupoids; the results of this article thus continue the author's research program, begun in $[4,5]$, to investigate the question of when a homotopy $\left\{\omega_{t}\right\}_{t \in[0,1]}$ of 2-cocycles on a groupoid $\mathcal{G}$ induces an isomorphism

$$
K_{*}\left(C^{*}\left(\mathcal{G}, \omega_{0}\right)\right) \cong K_{*}\left(C^{*}\left(\mathcal{G}, \omega_{1}\right)\right)
$$

of the $K$-theory groups of the twisted groupoid $C^{*}$-algebras. This question was inspired by the realization that Bott periodicity and the noncommutative tori can both be viewed as examples of a $K$-theoretic isomorphism arising from a homotopy of 2-cocycles. We hope that our investigation of the question of when a homotopy $\left\{\omega_{t}\right\}_{t \in[0,1]}$ of 2cocycles on a groupoid $\mathcal{G}$ induces the $K$-theoretic isomorphism (1.1) will increase our understanding of the structure of (twisted) groupoid $C^{*}$ algebras, as well as shed light on questions in $C^{*}$-algebraic classification and string theory. 
The study of full and reduced $C^{*}$-algebras $C^{*}(\mathcal{G}), C_{r}^{*}(\mathcal{G})$ associated to a locally compact groupoid $\mathcal{G}$ was initiated by Renault [13] and has since been actively pursued by many researchers. Although Renault also defined the twisted groupoid $C^{*}$-algebras $C^{*}(\mathcal{G}, \omega), C_{r}^{*}(\mathcal{G}, \omega)$ for a 2-cocycle $\omega \in Z^{2}(\mathcal{G}, \mathbb{T})[\mathbf{1 3}]$, these objects have received relatively little attention until recently. However, it has now become clear that twisted groupoid $C^{*}$-algebras can help answer many questions about the structure of untwisted groupoid $C^{*}$-algebras (cf. $\left.[\mathbf{2}, \mathbf{3}, \mathbf{7}, \mathbf{9}, \mathbf{1 0}]\right)$, as well as classifying those $C^{*}$-algebras which admit diagonal subalgebras (also known as Cartan subalgebras), cf., [8]. In another direction, [15] shows how the $K$-theory of twisted groupoid $C^{*}$-algebras classifies $D$ brane charges in many flavors of string theory.

1.1. Outline. In addition to its philosophical links with $[4,5]$, an attentive reader will notice similarities between the proofs presented in this article and several main results from $[4,5]$. To be precise, we begin this article by following the outline of the proof [4, Theorem 3.3] to calculate the $C_{0}(M)$-algebra structure associated to a locally trivial bundle of groups $\pi: \mathcal{G} \rightarrow M$. Then we use the results of this calculation, together with [5, Theorem 5.1] and a Mayer-Vietoris argument, to establish our main result in Theorem 3.3; Corollary 3.4 follows immediately.

2. $C_{0}(M)$-algebra structure. In this section, we describe the natural $C_{0}(M)$-algebra structure on $C^{*}(\mathcal{G}, \omega)$, where $\pi: \mathcal{G} \rightarrow M$ is a locally trivial bundle of groups over a locally compact Hausdorff space $M$, and $\omega$ is a 2-cocycle on $\mathcal{G}$. In order to state our results more precisely, we begin with some definitions.

We note that the following definition is non-standard in its requirement of local triviality; however, this hypothesis is necessary for the proofs of our later results and is satisfied by our motivating example of a vector bundle.

Definition 2.1. An (amenable) group bundle is a locally compact Hausdorff space $\mathcal{G}$ together with a continuous, open surjection $\pi: \mathcal{G} \rightarrow$ $M$ onto a locally compact Hausdorff space $M$ such that $\mathcal{G}_{m}:=\pi^{-1}(m)$ is isomorphic to a fixed (amenable) group $G$ for every $m \in M$, and such 
that $\mathcal{G}$ is locally trivial: for every point $m \in M$, there exists an open neighborhood $U$ of $m$ such that $\pi^{-1}(U)$ is homeomorphic to $U \times G$.

Given a group bundle $\pi: \mathcal{G} \rightarrow M$, write

$$
\mathcal{G}^{(2)}=\{(x, y) \in \mathcal{G} \times \mathcal{G}: \pi(x)=\pi(y)\} .
$$

Note that $\mathcal{G}^{(2)} \subseteq \mathcal{G} \times \mathcal{G}$ is a closed subspace; we equip it with the subspace topology.

Due to the isomorphism $\phi_{m}: \mathcal{G}_{m} \rightarrow G$, if $(x, y) \in \mathcal{G}^{(2)}$, then there is a unique element $z$ in $\mathcal{G}_{m}$ such that $\phi_{m}(z)=\phi_{m}(x) \phi_{m}(y)$. We will usually write $x y$ for this element. Similarly, for each $x \in \mathcal{G}_{m}$, there is a unique element $x^{-1} \in \mathcal{G}_{m}$ such that $x x^{-1}=x^{-1} x=\phi_{m}^{-1}(e)$. Moreover, the local triviality of $\mathcal{G}$ implies, in particular, that $\phi_{m}: \mathcal{G}_{m} \rightarrow G$ is a homeomorphism for all $m \in M$. As a consequence, the map $(x, y) \mapsto x y$ is continuous as a map $\mathcal{G}^{(2)} \rightarrow \mathcal{G}$, and $x \mapsto x^{-1}$ is a continuous map from $\mathcal{G}$ to itself.

Let $C_{c}(\mathcal{G})$ denote the collection of those continuous complex-valued functions $f$ on the total space $\mathcal{G}$ of the group bundle such that $\operatorname{supp} f$ is compact, and let $\lambda$ be a fixed Haar measure on the fiber group $G \cong \mathcal{G}_{m}$ of the group bundle. The local triviality of $\mathcal{G}$ then implies the following proposition.

Proposition 2.2. Let $f \in C_{c}(\mathcal{G})$. Then the function

$$
m \longmapsto \int_{\mathcal{G}_{m}} f(x) d \lambda(x)
$$

lies in $C_{0}(M)$.

Proof. We begin by observing that, since the Haar measure is, in particular, a Radon measure, and $\operatorname{supp} f$ is compact, we know that $L_{m}:=\lambda\left(\mathcal{G}_{m} \cap \operatorname{supp} f\right)$ is finite for each $m \in M$.

Fix $m \in M$, and let $U$ be a neighborhood of $m$ such that $\left.\mathcal{G}\right|_{U} \cong$ $U \times G$. Due to this isomorphism, we will write $f(n, g)$ rather than $f(x)$ for $\left.x \in \mathcal{G}\right|_{U}$. Since $\operatorname{supp} f$ is compact, $\pi_{2}(\operatorname{supp} f \cap(U \times G)) \subseteq G$ is also compact; consequently, for any $\epsilon>0$, we can find a smaller neighborhood $U_{\epsilon} \subseteq U$ of $m$ such that, if $n \in U_{\epsilon}$,

$$
|f(n, g)-f(m, g)|<\epsilon / L_{m} \quad \text { for all } g \in G .
$$


It follows that, if $n \in U_{\epsilon}$,

$$
\begin{aligned}
& \mid \int_{\mathcal{G}_{n}} f(n, g) d \lambda(g)-\int_{\mathcal{G}_{m}} f(m, g) d \lambda(g) \mid \\
& \leq \int_{G}|f(n, g)-f(m, g)| d \lambda(g)<\epsilon .
\end{aligned}
$$

In other words,

$$
m \longmapsto \int_{\mathcal{G}_{m}} f(x) d \lambda(x) \in C_{0}(M),
$$

as claimed.

Definition 2.3. A 2-cocycle on $\mathcal{G}$ is a continuous circle-valued function $\omega: \mathcal{G}^{(2)} \rightarrow \mathbb{T}$ such that, whenever $(x, y),(y, z) \in \mathcal{G}^{(2)}$, the cocycle condition holds:

$$
\omega(x y, z) \omega(x, y)=\omega(x, y z) \omega(y, z) .
$$

\section{Example 2.4.}

(i) For any group bundle $\mathcal{G}$, the function $\omega: \mathcal{G}^{(2)} \rightarrow \mathbb{T}$ given by $\omega(x, y)=1$ for all $(x, y) \in \mathcal{G}^{(2)}$ is a 2-cocycle on $\mathcal{G}$, called the trivial 2-cocycle.

(ii) As discussed in the introduction, if $\pi: V \rightarrow M$ is a vector bundle and $\sigma: V^{(2)} \rightarrow \mathbb{R}$ is a 2-form on $V$, then $\omega(v, w):=e^{2 \pi i \sigma(v, w)}$ defines a 2-cocycle on $V$.

A 2-cocycle on $\mathcal{G}$ allows us to define a twisted convolution multiplication on $\mathcal{G}$, which in turn will allow us to build the associated twisted $C^{*}$-algebra $C^{*}(\mathcal{G}, \omega)$. This is a particular case of the construction of a twisted groupoid $C^{*}$-algebra, as described in [13, Chapter II].

Given a 2-cocycle $\omega$ on $\mathcal{G}$ and $f, g \in C_{c}(\mathcal{G})$, we define the twisted convolution product of $f$ and $g$ by

$$
f * g(x):=\int_{\mathcal{G}_{\pi(x)}} f(x y) g\left(y^{-1}\right) \omega\left(x y, y^{-1}\right) d \lambda(y) .
$$

We also define an involution on $C_{c}(\mathcal{G})$ that incorporates the 2-cocycle:

$$
f^{*}(x):=\overline{f\left(x^{-1}\right) \omega\left(x, x^{-1}\right)} .
$$


Renault proved [13, Proposition II.1.1] that the multiplication is well defined (that is, that $f * g \in C_{c}(\mathcal{G})$ as claimed) and associative, and that $\left(f^{*}\right)^{*}=f$ so that the involution is involutive. (The proof of associativity relies on the cocycle condition (2.1).)

Thus, we have a $*$-algebra structure on $C_{c}(\mathcal{G})$. To indicate the importance of the 2-cocycle in this structure, we will often write $C_{c}(\mathcal{G}, \omega)$ for this *-algebra. To avoid confusion between the use of * to indicate multiplication and the involution, we will usually denote the multiplication in $C_{c}(\mathcal{G}, \omega)$ by juxtaposition: $f g:=f * g$.

The twisted $C^{*}$-algebra $C^{*}(\mathcal{G}, \omega)$ is the completion of $C_{c}(\mathcal{G}, \omega)$ with respect to the full or universal $C^{*}$-norm (2.2). In order to give the precise definition of the universal norm, we require some preliminary definitions.

Definition 2.5. Let $\mathcal{H}$ be a Hilbert space. We say that a $*$ homomorphism $\pi: C_{c}(\mathcal{G}, \omega) \rightarrow B(\mathcal{H})$ is a representation of $C_{c}(\mathcal{G}, \omega)$ if it is nondegenerate in the sense that

$$
\overline{\operatorname{span}}\left\{\pi(f) \xi: f \in C_{c}(\mathcal{G}, \omega), \xi \in \mathcal{H}\right\}=\mathcal{H} .
$$

Definition 2.6. The $I$-norm on $C_{c}(\mathcal{G})$ is given by

$$
\|f\|_{I}=\max \left\{\sup _{m \in M} \int_{\mathcal{G}_{m}}|f(x)| d \lambda(x), \sup _{m \in M} \int_{\mathcal{G}_{m}}\left|f\left(x^{-1}\right)\right| d \lambda(x)\right\} .
$$

We say that a representation $\phi$ of $C_{c}(\mathcal{G})$ is $I$-norm-bounded if $\|\phi(f)\| \leq$ $\|f\|_{I}$ for any $f \in C_{c}(G)$.

Then, [13, Proposition II.1.11] combines with the comments following [13, Definition II.1.5] to tell us that

$$
\begin{aligned}
\|f\|:=\sup \{\|\phi(f)\|: \phi & \text { is an } I \text {-norm-bounded } \\
& \text { representation of } \left.C_{c}(\mathcal{G}, \omega)\right\}
\end{aligned}
$$

is a $C^{*}$-norm.

Definition 2.7 (cf., [13, Definition II.1.12]). The (universal) twisted $C^{*}$-algebra of $\mathcal{G}$, denoted $C^{*}(\mathcal{G}, \omega)$, is the completion of $C_{c}(\mathcal{G}, \omega)$ with respect to the norm $(2.2)$. 
The goal of this section is Proposition 2.9, in which we prove that, despite this intricate definition of the norm on $C^{*}(\mathcal{G}, \omega)$, this $C^{*}$-algebra admits a $C_{0}(M)$-algebra structure which makes it much more tractable.

Definition 2.8. Let $A$ be a $C^{*}$-algebra and $M$ a locally compact Hausdorff space. We say that $A$ is a $C_{0}(M)$-algebra if there exists a $*$-homomorphism

$$
\Phi: C_{0}(M) \rightarrow Z M(A) .
$$

Given $f \in C_{0}(M), a \in A$, we will usually write $f \cdot a$ for $\Phi(f) a$.

A $C_{0}(M)$-algebra fibers over $M$ in a natural way. If $m \in M$, let

$$
I_{m}:=\overline{C_{0}(M \backslash\{m\}) \cdot A} ;
$$

then $I_{m}$ is an ideal, and the quotient $A_{m}:=A / I_{m}$ gives the fiber of $A$ at $m$. Indeed, [17, Theorem C.26] tells us that there is a unique topology on the bundle $\mathcal{A}:=\coprod_{m \in M} A_{m}$ such that $A=\Gamma_{0}(\mathcal{A})$ are the continuous sections of $\mathcal{A}$ that vanish at infinity.

In an analogous manner to the construction of $A_{m}$, for any $C_{0}(M)$ algebra $A$ and any closed subset $F \subseteq M$, we have a quotient $A_{F}$ of $A$ :

$$
A_{F}:=A / I_{F} \quad \text { where } \quad I_{F}:=\overline{C_{0}(M \backslash F) \cdot A} .
$$

Proposition 2.9 describes the $C_{0}(M)$-algebra structure carried by the twisted $C^{*}$-algebra of a group bundle $\pi: \mathcal{G} \rightarrow M$. A similar result is obtained by Goehle for crossed products by a group bundle in [6, Proposition 1.2 and Lemma 1.4], and the proof of Proposition 2.9 below proceeds along similar lines to Goehle's proof, as well as to the proof of $[4$, Theorem 3.5].

Proposition 2.9. Let $\pi: \mathcal{G} \rightarrow M$ be a group bundle, and let $\omega$ be a 2 -cocycle on $\mathcal{G}$. Then $C^{*}(\mathcal{G}, \omega)$ is a $C_{0}(M)$-algebra, with $C^{*}(\mathcal{G}, \omega)_{F} \cong$ $C^{*}\left(\left.\mathcal{G}\right|_{F}, \omega\right)$ for any closed $F \subseteq M$.

Proof. The action of $C_{0}(M)$ on $C^{*}(\mathcal{G}, \omega)$ is defined as one might expect: for $\phi \in C_{0}(M), f \in C_{c}(\mathcal{G}, \omega)$, define $\phi \cdot f \in C_{c}(\mathcal{G}, \omega)$ by

$$
\phi \cdot f(x)=\phi(\pi(x)) f(x) .
$$


It is immediate that the action is linear and multiplicative; because $\pi(x)=\pi(y)$ whenever $x$ and $y$ are in the same fiber of the group bundle, and in particular, we have $\pi(x)=\pi\left(x^{-1}\right)$, we also have

$$
f *(\phi \cdot g)=(\phi \cdot f) * g=\phi \cdot(f * g)
$$

for any $f, g \in C_{c}(\mathcal{G}, \omega)$ and any $\phi \in C_{0}(M)$. In other words, $C_{0}(M)$ acts centrally on $C_{c}(\mathcal{G}, \omega)$. Moreover, a straightforward check shows that $(\phi \cdot f)^{*}=\phi^{*} \cdot f^{*}$, so the centrality of the action implies that it is also $*$-preserving.

Thus, to see that this action gives rise to a $*$-homomorphism $\Phi$ : $C_{0}(M) \rightarrow Z M\left(C^{*}(\mathcal{G}, \omega)\right)$, we merely need to check that the action is bounded, that is, we will show that $\|\phi \cdot f\| \leq\|\phi\|_{\infty}\|f\|$ for any $f \in C_{c}(\mathcal{G}, \omega)$ and any $\phi \in C_{0}(M)$.

Fix $\phi \in C_{0}(M), f \in C_{c}(\mathcal{G}, \omega)$. Letting $K_{f}=\operatorname{supp} f$, choose $\phi_{f} \in C_{0}(M)$ to be 1 on $\pi\left(K_{f}\right)$. Then the function,

$$
\xi(m):=\left(\|\phi\|_{\infty}^{2}-|\phi(m)|^{2}\right)\left|\phi_{f}(m)\right|^{2},
$$

is in $C_{0}(M)$. Moreover, $\xi$ is positive and hence has a positive square root, $k$. The positivity of $k$, combined with our earlier observations that the action is multiplicative, $*$-preserving, and central, means that

$$
(\xi \cdot f)^{*} f=k^{2} \cdot\left(f^{*} f\right)=(k \cdot f)^{*}(k \cdot f) .
$$

Therefore, $(\xi \cdot f)^{*} f \geq 0$ in $C^{*}(\mathcal{G}, \omega)$. Since $\xi^{*}=\xi$, this inequality tells us that

$$
\begin{aligned}
& 0 \leq(\xi \cdot f)^{*} f=\xi \cdot\left(f^{*} f\right)=\left(\|\phi\|_{\infty}^{2}\left|\phi_{f}\right|^{2}\right) \\
& \cdot\left(f^{*} f\right)-\left(\left|\phi \cdot \phi_{f}\right|^{2}\right) \cdot\left(f^{*} f\right) .
\end{aligned}
$$

Since positivity preserves norms, it follows that

$$
\left\|\left(\left|\phi \cdot \phi_{f}\right|^{2}\right) \cdot\left(f^{*} f\right)\right\| \leq\left\|\left(\|\phi\|_{\infty}^{2}\left|\phi_{f}\right|^{2}\right) \cdot\left(f^{*} f\right)\right\| .
$$

Observe that, since $\phi_{f}=1$ on $\operatorname{supp} f$, the function $\|\phi\|_{\infty}^{2}\left|\phi_{f}\right|^{2}$ acts on $f$ as multiplication by the constant $\|\phi\|_{\infty}^{2}$. Moreover,

$$
\left(\left|\phi \cdot \phi_{f}\right|^{2}\right) \cdot f^{*} f=\left(\left(\phi \cdot \phi_{f}\right) \cdot f\right)^{*}\left(\left(\phi \cdot \phi_{f}\right) \cdot f\right)=(\phi \cdot f)^{*}(\phi \cdot f),
$$

because the action is multiplicative, central and $*$-preserving. Thus, equation (2.4) becomes

$$
\left\|(\phi \cdot f)^{*}(\phi \cdot f)\right\| \leq\|\phi\|_{\infty}^{2}\left\|f^{*} f\right\|,
$$


so by the $C^{*}$-identity, we have

$$
\|\phi \cdot f\| \leq\|\phi\|_{\infty}\|f\|
$$

as claimed.

We have thus shown that the action is bounded, so it extends to a *-homomorphism $\Phi: C_{0}(M) \rightarrow M\left(C^{*}(\mathcal{G}, \omega)\right)$. In fact, $\operatorname{Im} \Phi \subseteq$ $Z M\left(C^{*}(\mathcal{G}, \omega)\right)$ because $\operatorname{Im} \Phi$ acts centrally on the dense $*$-subalgebra $C_{c}(\mathcal{G}, \omega)$. Moreover, $\Phi\left(\phi_{f}\right) f=f$ for any $f \in C_{c}(\mathcal{G}, \omega)$, so $\Phi\left(C_{0}(M)\right)$. $C^{*}(\mathcal{G}, \omega)$ contains the dense subalgebra $C_{c}(\mathcal{G}, \omega)$. Consequently,

$$
\overline{\Phi\left(C_{0}(M)\right) \cdot C^{*}(\mathcal{G}, \omega)}=C^{*}(\mathcal{G}, \omega) .
$$

In other words, $\Phi$ makes $C^{*}(\mathcal{G}, \omega)$ into a $C_{0}(M)$-algebra as claimed. We will use this action of $C_{0}(M)$ on $C^{*}(\mathcal{G}, \omega)$ throughout the rest of this proof, usually denoting it by $\phi \cdot f$ as above rather than by $\Phi$.

Checking that

$$
C^{*}(\mathcal{G}, \omega)_{F} \cong C^{*}\left(\left.\mathcal{G}\right|_{F}, \omega\right)
$$

for any closed subset $F \subseteq M$ as claimed will require rather more work.

Recall that $C^{*}(\mathcal{G}, \omega)_{F}$ is given by the quotient $C^{*}(\mathcal{G}, \omega) / I_{F}$, where

$$
I_{F}:=\overline{\operatorname{span}}\left\{\phi \cdot f: \phi \in C_{0}(M \backslash F), f \in C^{*}(\mathcal{G}, \omega)\right\} .
$$

Thus, in order to prove Proposition 2.9, we must show that

$$
C^{*}\left(\left.\mathcal{G}\right|_{F}, \omega\right) \cong C^{*}(\mathcal{G}, \omega) / I_{F}
$$

We will begin by showing that we can indeed exhibit $C^{*}\left(\left.\mathcal{G}\right|_{F}, \omega\right)$ as a quotient of $C^{*}(\mathcal{G}, \omega)$ whenever $F \subseteq M$ is closed.

Fix a closed subset $F \subseteq M$, and let $q_{F}: C_{c}(\mathcal{G}, \omega) \rightarrow C_{c}\left(\left.\mathcal{G}\right|_{F}, \omega\right)$ be the restriction map. By the definition of the $I$-norm given in Definition 2.6, $q_{F}$ is $I$-norm-bounded; since the operations in the $*$-algebra $C_{c}(\mathcal{G}, \omega)$ respect the way $\mathcal{G}$ fibers over $M, q_{F}$ is also a $*$-homomorphism. Consequently, for any $I$-norm-bounded representation $\psi$ of $C_{c}\left(\left.\mathcal{G}\right|_{F}, \omega\right)$, the composition $\psi \circ q_{F}$ is an $I$-norm-bounded representation of $C_{c}(\mathcal{G}, \omega)$. 
Thus, for any $f \in C_{c}(\mathcal{G}, \omega)$,

$$
\begin{aligned}
\left\|q_{F}(f)\right\|= & \sup \left\{\left\|\psi \circ q_{F}(f)\right\|:\right. \\
& \left.\psi \text { an } I \text {-norm-bounded representation of } C_{c}\left(\mathcal{G}_{F}, \omega\right)\right\} \\
\leq & \sup \{\|\Psi(f)\|: \Psi \text { an } I \text {-norm-bounded representation of } \\
= & \left.C_{c}(\mathcal{G}, \omega)\right\}
\end{aligned}
$$

Hence, $q_{F}$ extends to a $*$-homomorphism, also denoted $q_{F}$, from $C^{*}(\mathcal{G}, \omega)$ to $C^{*}\left(\left.\mathcal{G}\right|_{F}, \omega\right)$.

Note that any function $f \in C_{c}\left(\left.\mathcal{G}\right|_{F}\right)$ can be extended to $\bar{f} \in C_{c}(\mathcal{G})$ by the Tietze extension theorem, so that $q_{F}(\bar{f})=f$. Since $C_{c}\left(\left.\mathcal{G}\right|_{F}\right) \subseteq$ $C^{*}\left(\left.\mathcal{G}\right|_{F}, \omega\right)$ is dense, this implies that $q_{F}: C^{*}(\mathcal{G}, \omega) \rightarrow C^{*}\left(\left.\mathcal{G}\right|_{F}, \omega\right)$ is surjective. In other words,

$$
C^{*}\left(\left.\mathcal{G}\right|_{F}, \omega\right) \cong C^{*}(\mathcal{G}, \omega) / \operatorname{ker} q_{F} .
$$

Thus, to see that $C^{*}\left(\left.\mathcal{G}\right|_{F}, \omega\right)=C^{*}(\mathcal{G}, \omega)_{F}$, it suffices to show that $\operatorname{ker} q_{F}=I_{F}$. A standard approximation argument will show that $\operatorname{ker} q_{F} \supseteq I_{F}$; the tricky part is showing that $\operatorname{ker} q_{F} \subseteq I_{F}$.

To show that $\operatorname{ker} q_{F} \subseteq I_{F}$, we will show that any representation $L$ of $C_{c}(\mathcal{G}, \omega)$ such that $L\left(I_{F}\right)=0$ must factor through $q_{F}$, so that we can write $L=L^{\prime} \circ q_{F}$ for some $I$-norm-bounded representation $L^{\prime}$ of $C_{C}\left(\left.\mathcal{G}\right|_{F}, \omega\right)$. This will imply that $\operatorname{ker} q_{F} \subseteq \operatorname{ker} L$ for all such representations $L$, and consequently that ker $q_{F} \subseteq I_{F}$ as desired.

Given an $I$-norm-bounded representation $L: C_{c}(\mathcal{G}, \omega) \rightarrow B(\mathcal{H})$ such that $L\left(I_{F}\right)=0$, define $L^{\prime}: C_{c}\left(\left.\mathcal{G}\right|_{F}, \omega\right) \rightarrow B(\mathcal{H})$ by

$$
L^{\prime}\left(q_{F}(f)\right):=L(f) .
$$

We would like to show that $L^{\prime}$ is a representation of $C_{c}\left(\left.\mathcal{G}\right|_{F}, \omega\right)$. Note that $L^{\prime}$ preserves the *-algebra structure on $C_{c}\left(\left.\mathcal{G}\right|_{F}, \omega\right)$ because $L$ and $q_{F}$ do so, since they are $*$-homomorphisms. Moreover, $L^{\prime}$ is nondegenerate because $L$ is and because $q_{F}: C_{c}(\mathcal{G}, \omega) \rightarrow C_{c}\left(\left.\mathcal{G}\right|_{F}, \omega\right)$ is surjective. Thus, we only need to check that $L^{\prime}$ is well defined and bounded.

To see that $L^{\prime}$ is well defined, we need to show that $L(f)=L(g)$ whenever $q_{F}(f)=q_{F}(g)$. 
Lemma 2.10. If $f, g \in C_{c}(\mathcal{G}, \omega)$ satisfy $q_{F}(f)=q_{F}(g)$, then the function,

$$
h=f-g \in C_{c}(\mathcal{G}, \omega),
$$

lies in $I_{F}$. Consequently, $L(f)=L(g)$, and $L^{\prime}$ is well defined on $C_{c}\left(\left.\mathcal{G}\right|_{F}, \omega\right)$.

Proof. Let $\left\{f_{K, U}\right\}_{K, U}$ be an approximate unit for $C_{0}(M \backslash F)$, indexed by pairs $(K, U)$ where $K \subseteq M$ is compact and $U \supseteq F$ is open, such that $U \cup K=M ; f_{K, U}$ is 1 on $K \backslash U$ and 0 on $F$; and $0 \leq f_{K, U}(m) \leq 1$ for all $m \in M$. (We can always construct such functions by using Urysohn's lemma.)

We will show that the $I$-norm,

$$
\left\|h-f_{K, U} \cdot h\right\|_{I} \longrightarrow 0,
$$

takes the limit over increasing $K$ and decreasing $U$. Since the norm in $C^{*}(\mathcal{G}, \omega)$ is dominated by the $I$-norm, it will follow that

$$
h=\lim _{K, U} f_{K, U} \cdot h
$$

in $C^{*}(\mathcal{G}, \omega)$, and consequently, $h \in I_{F}$.

We first observe that the function,

$$
m \longmapsto \lambda\left(\mathcal{G}_{m} \cap \operatorname{supp} h\right),
$$

is bounded, where $\lambda$ denotes our chosen Haar measure on the fiber group $\mathcal{G}_{m} \cong G$ (recall that $\mathcal{G}_{m} \cong \mathcal{G}_{n} \cong G$ for all $m, n \in M$ ). To see that this function is bounded, let $W$ be an open neighborhood of $\operatorname{supp} h$, and use Urysohn's lemma to construct $k_{W} \in C_{c}(\mathcal{G})$ such that $\left.k_{W}\right|_{\operatorname{supp} h}=1$ and $\operatorname{supp} k_{W} \subseteq W$. Then, for any $m \in M$,

$$
\int_{\mathcal{G}_{m}} k_{W}(x) d \lambda(x) \geq \lambda\left(\mathcal{G}_{m} \cap \operatorname{supp} h\right) .
$$

Moreover, we know from Proposition 2.2 that

$$
m \longmapsto \int_{\mathcal{G}_{m}} k_{W}(x) d \lambda(x) \in C_{0}(M),
$$

because $k_{W} \in C_{c}(\mathcal{G})$. Since this function is an upper bound for the function $m \mapsto \lambda\left(\mathcal{G}_{m} \cap \operatorname{supp} h\right)$, it follows that

$$
m \longmapsto \lambda\left(\mathcal{G}_{m} \cap \operatorname{supp} h\right)
$$


is bounded on $M$, as claimed. Let $\ell$ be the maximum value of the function $m \mapsto \lambda\left(\mathcal{G}_{m} \cap \operatorname{supp} h\right)$.

Let $\epsilon>0$ be given, and let $U=\pi\left(h^{-1}\left(B_{\epsilon / 2 \ell}(0)\right)\right)$. Then $U \subseteq M$ is open and contains $F$. Let

$$
K=\pi(\operatorname{supp} h) \subseteq M ;
$$

we will show that, for any $\left(K^{\prime}, U^{\prime}\right) \geq(K, U)$, we have

$$
\left\|h-f_{K^{\prime}, U^{\prime}} \cdot h\right\|_{I}<\epsilon .
$$

Recall that

$$
\begin{aligned}
\left\|h-f_{K^{\prime}, U^{\prime}} \cdot h\right\|_{I}=\max \{ & \sup _{m \in M} \int_{\mathcal{G}_{m}}\left|h(x)-f_{K^{\prime}, U^{\prime}}(m) h(x)\right| d \lambda(x), \\
& \left.\sup _{m \in M} \int_{\mathcal{G}_{m}}\left|h\left(x^{-1}\right)-f_{K^{\prime}, U^{\prime}}(m) h\left(x^{-1}\right)\right| d \lambda(x)\right\} .
\end{aligned}
$$

If $m \in K^{\prime} \backslash U^{\prime}$, then $f_{K^{\prime}, U^{\prime}}(m)=1$ and the above integrals are zero. If $m \in U^{\prime}$, then since $\left(K^{\prime}, U^{\prime}\right) \geq(K, U)$, we also have $m \in U$, so

$$
|h(x)|<\epsilon / 2 \ell \text { for all } x \in \mathcal{G}_{m} .
$$

Moreover, the fact that $0 \leq f_{K^{\prime}, U^{\prime}}(m) \leq 1$ for all $m \in M$ implies that, for any $x \in \mathcal{G}$,

$$
\left|h(x)-f_{K^{\prime}, U^{\prime}}(\pi(x)) h(x)\right| \leq 2|h(x)| .
$$

It follows that

$$
\begin{aligned}
&\left\|h-f_{K^{\prime}, U^{\prime}} \cdot h\right\|_{I} \leq \max \left\{\sup _{m \in U^{\prime}} \int_{\mathcal{G}_{m}}\left|h(x)-f_{K^{\prime}, U^{\prime}}(m) h(x)\right| d \lambda(x),\right. \\
& \leq \max \left\{\sup _{m \in U^{\prime}} \int_{m \in U^{\prime}} \int_{\mathcal{G}_{m}}\left|h\left(x^{-1}\right)-f_{K^{\prime}, U^{\prime}}(m) h\left(x^{-1}\right)\right| d \lambda(x)\right\} \\
&\left.\sup _{m \in U^{\prime}} \int_{\mathcal{G}_{m}} 2\left|h\left(x^{-1}\right)\right| d \lambda(x)\right\} \\
& \leq 2 \sup _{m \in U^{\prime}} \sup _{x \in \mathcal{G}_{m}}|h(x)| \lambda\left(\mathcal{G}_{m} \cap \operatorname{supp} h\right) \\
&<\epsilon .
\end{aligned}
$$


Consequently,

$$
\lim _{K, U}\left\|h-f_{K, U} \cdot h\right\|_{I}=0 .
$$

It follows that $h \in I_{F}$, as claimed, and so $L(h)=0$. This proves that $L^{\prime}$ is well defined.

Since $L^{\prime}$ is well defined, we proceed to show that $\left\|L^{\prime}(f)\right\| \leq\|f\|_{I}$ for any $f \in C_{c}\left(\left.\mathcal{G}\right|_{F}, \omega\right)$.

First, we note that Proposition 2.2 and the definition of the $I$ norm imply that the function $m \mapsto\left\|q_{\{m\}}(f)\right\|_{I}$ is continuous for each $f \in C_{c}(\mathcal{G})$. Consequently, if we fix $f \in C_{c}(\mathcal{G}, \omega), \epsilon>0$, the set

$$
W_{\epsilon}=\left\{m \in M:\left\|q_{\{m\}}(f)\right\|_{I}<\left\|q_{F}(f)\right\|_{I}+\epsilon\right\}
$$

is open; note that $F \subseteq W_{\epsilon}$. Thus, we can choose $\psi_{f, \epsilon} \in C_{0}(M)$ such that $0 \leq \psi_{f, \epsilon}(m) \leq 1$ for all $m \in M ; \psi_{f, \epsilon}=1$ on $F ; \psi_{f, \epsilon}=0$ off $W_{\epsilon}$. Since $\psi_{f, \epsilon}=1$ on $F$, we have

$$
L(f)=L^{\prime}\left(q_{F}(f)\right)=L^{\prime}\left(q_{F}\left(\psi_{f, \epsilon} \cdot f\right)\right)=L\left(\psi_{f, \epsilon} \cdot f\right) .
$$

Consequently,

$$
\begin{aligned}
\left\|L^{\prime}\left(q_{F}(f)\right)\right\| & =\left\|L\left(\psi_{f, \epsilon} \cdot f\right)\right\| \leq\left\|\psi_{f, \epsilon} \cdot f\right\|_{I} \\
& =\max \left\{\sup _{m \in M} \int_{\mathcal{G}_{m}}\left|\psi_{f, \epsilon}(m) f(x)\right| d \lambda(x),\right. \\
& \left.\leq \sup _{m \in M} \int_{\mathcal{G}_{m}}\left|\psi_{f, \epsilon}(m) f\left(x^{-1}\right)\right| d \lambda(x)\right\} \\
& \max \left\{\sup _{m \in W_{\epsilon}} \int_{\mathcal{G}_{m}}|f(x)| d \lambda(x),\right. \\
& \left.=\sup _{m \in W_{\epsilon}} \int_{\mathcal{G}_{m}}\left|f\left(x^{-1}\right)\right| d \lambda(x)\right\} \\
& <\left\|q_{\{m\}}(f)\right\|_{I}(f) \|_{I}+\epsilon .
\end{aligned}
$$

Since we can choose such a $\psi_{f, \epsilon}$ for any $\epsilon>0$, it follows that

$$
\left\|L^{\prime}\left(q_{F}(f)\right)\right\| \leq\left\|q_{F}(f)\right\|_{I} .
$$

The fact that $q_{F}: C_{c}(\mathcal{G}, \omega) \rightarrow C_{c}\left(\left.\mathcal{G}\right|_{F}, \omega\right)$ is onto now implies that $L^{\prime}$ is an $I$-norm-bounded representation of $C_{c}\left(\left.\mathcal{G}\right|_{F}, \omega\right)$. 
In other words, every representation of $C_{c}(\mathcal{G}, \omega)$ which kills $I_{F}$ also factors through $q_{F}$, so $\operatorname{ker} q_{F}=I_{F}$ as claimed, that is,

$$
C^{*}\left(\left.\mathcal{G}\right|_{F}, \omega\right) \cong C^{*}(\mathcal{G}, \omega)_{F},
$$

for any $F \subseteq M$ closed. This finishes the proof of Proposition 2.9.

Knowing that $C^{*}(\mathcal{G}, \omega)_{F}=C^{*}\left(\left.\mathcal{G}\right|_{F}, \omega\right)$ will be crucial for the arguments in the next section. However, we will also need a result (Proposition 2.12) about the way ideals in $C_{0}(M)$-algebras relate. Although this result is undoubtedly well known to experts, we include a proof for completeness.

We begin with an observation about approximate units in $C_{0}(M)$. Since $M$ is locally compact Hausdorff, for any closed set $F \subseteq M$, we can write $M \backslash F$ as an increasing union $M \backslash F=\cup_{i \in I} K_{i}$ of compact sets, and then Urysohn's lemma tells us that we can find an approximate unit $\left\{\phi_{i}^{F}\right\}_{i \in I}$ for $C_{0}(M \backslash F)$ such that $\phi_{i}^{F}$ is 1 on $K_{i}$. It follows that, for any $m \in M \backslash F$, there exists $J \in I$ such that $i \geq J$ implies $\phi_{i}^{F}(m)=1$.

Lemma 2.11. Let $A$ be a $C_{0}(M)$-algebra for a second countable locally compact Hausdorff space $M$, and let $F_{1}, F_{2} \subseteq M$ be closed. For any $a \in I_{F_{1} \cap F_{2}}$, we can find $g \in I_{F_{1}}$ and $h \in I_{F_{2}}$ such that $a-g-h \in I_{F_{1} \cup F_{2}}$.

Proof. Let $\left\{\phi_{i}^{12}\right\}_{i \in I}$ denote the approximate unit for

$$
C_{0}\left(M \backslash\left(F_{1} \cap F_{2}\right)\right)
$$

described above. Then, given $\epsilon>0$ and $a \in I_{F_{1} \cap F_{2}}$, there exists $J$ such that $\left\|a-\phi_{J}^{12} \cdot a\right\|<\epsilon$. Let $\left\{\phi_{\lambda}^{1}\right\}_{\lambda \in \Lambda}$ and $\left\{\phi_{\mu}^{2}\right\}_{\mu \in S}$ be the analogous approximate units for $C_{0}\left(M \backslash F_{1}\right)$ and $C_{0}\left(M \backslash F_{2}\right)$, respectively. Then

$$
\begin{aligned}
& g_{\epsilon}:=\left(\lim _{\lambda} \phi_{\lambda}^{1} \phi_{J}^{12}\right) \cdot a \in I_{F_{1}}, \\
& h_{\epsilon}:=\left(\lim _{\mu} \phi_{\mu}^{2} \phi_{J}\right) \cdot a \in I_{F_{2}} .
\end{aligned}
$$

Moreover, $\phi_{J}^{12} \cdot a-g_{\epsilon}-h_{\epsilon} \in I_{F_{1} \cup F_{2}}$. To see this, set $m \in F_{1} \cup F_{2}$. If $m \in F_{1} \backslash F_{2}$, we can choose $L$ large enough that $\mu \geq L$ implies $\phi_{\mu}^{2}(m)=1$; consequently,

$$
\phi_{J}^{12}(m)-\phi_{\lambda}^{1}(m) \phi_{J}^{12}(m)-\phi_{\mu}^{2}(m) \phi_{J}^{12}(m)=0,
$$


since $\phi_{\lambda}^{1}(m)=0$ for any $\lambda$ when $m \in F_{1}$. Similarly, if $m \in F_{2} \backslash F_{1}$, we can choose $K$ such that $\lambda \geq K$ implies $\phi_{\lambda}^{1}(m)=1$, and so equation (2.5) also holds; and if $m \in F_{1} \cap F_{2}$, then $\phi_{J}^{12}(m)=0$ and equation (2.5) still holds. Thus, if $\lambda, \mu$ are large enough, then equation (2.5) holds for all $m \in F_{1} \cup F_{2}$, and taking the limit over $\lambda, \mu$ reveals that

$$
\phi_{J}^{12} \cdot a-g_{\epsilon}-h_{\epsilon}=\left(\phi_{J}^{12}-\lim _{\lambda} \phi_{\lambda}^{1} \phi_{J}^{12}-\lim _{\mu} \phi_{\mu}^{2} \phi_{J}^{12}\right) \cdot a \in I_{F_{1} \cup F_{2}},
$$

as claimed.

Therefore, denoting the norm in the quotient $A_{F}$ by $\|\cdot\|_{F}$, we see that

$$
\begin{aligned}
\left\|a-g_{\epsilon}-h_{\epsilon}\right\|_{F_{1} \cup F_{2}} & \leq\left\|a-\phi_{J}^{12} \cdot a\right\|_{F_{1} \cup F_{2}}+\left\|\phi_{J}^{12} \cdot a-g_{\epsilon}-h_{\epsilon}\right\|_{F_{1} \cup F_{2}} \\
& =\left\|a-\phi_{J}^{12} \cdot a\right\|_{F_{1} \cup F_{2}} \\
& \leq\left\|a-\phi_{J}^{12} \cdot a\right\| \\
& <\epsilon .
\end{aligned}
$$

Furthermore, since $0 \leq \phi_{\lambda}^{1}(m) \leq 1$ for all $m \in M$,

$$
\left\|g_{\epsilon}-g_{\epsilon^{\prime}}\right\|=\left\|\lim _{\lambda} \phi_{\lambda}^{1}\left(\phi_{J}^{12}-\phi_{J^{\prime}}^{12}\right) \cdot a\right\| \leq\left\|\left(\phi_{J}^{12}-\phi_{J^{\prime}}^{12}\right) \cdot a\right\|<\epsilon+\epsilon^{\prime},
$$

so the net $\left\{g_{\epsilon}\right\}_{\epsilon>0}$ converges in $A$. The same argument will show that $\left\{h_{\epsilon}\right\}_{\epsilon>0}$ also converges. Setting $g:=\lim _{\epsilon} g_{\epsilon}$ and $h:=\lim _{\epsilon} h_{\epsilon}$, we have $g \in I_{F_{1}}$ since $g_{\epsilon} \in I_{F_{1}}$ for all $\epsilon$; similarly, $h \in I_{F_{2}}$. We claim that $a-g-h \in I_{F_{1} \cup F_{2}}$.

To see this, let $\delta>0$ be given, and suppose that $\epsilon$ is small enough such that $\left\|g-g_{\epsilon}\right\|,\left\|h-h_{\epsilon}\right\|<\delta$. Without loss of generality, suppose $\epsilon<\delta$. Then,

$$
\begin{aligned}
\|a-g-h\|_{F_{1} \cup F_{2}} & \leq\left\|a-g_{\epsilon}-h_{\epsilon}\right\|_{F_{1} \cup F_{2}}+\left\|g_{\epsilon}-g\right\|_{F_{1} \cup F_{2}}+\left\|h_{\epsilon}-h\right\|_{F_{1} \cup F_{2}} \\
& <\epsilon+2 \delta<3 \delta .
\end{aligned}
$$

It follows that $a-g-h \in I_{F_{1} \cup F_{2}}$, as claimed.

Proposition 2.12. Let $A$ be a $C_{0}(X)$-algebra for a locally compact Hausdorff space $X$, and let $F_{1}, F_{2} \subseteq M$ be closed. Then we have a short exact sequence of $C^{*}$-algebras

$$
0 \longrightarrow A_{F_{1} \cup F_{2}} \longrightarrow A_{F_{1}} \oplus A_{F_{2}} \longrightarrow A_{F_{1} \cap F_{2}} \longrightarrow 0 .
$$


Proof. We begin by showing that

$$
I_{F_{1} \cup F_{2}}:=\overline{C_{0}\left(M \backslash\left(F_{1} \cup F_{2}\right)\right) \cdot A}=I_{F_{1}} \cap I_{F_{2}}
$$

is contained in both $I_{F_{1}}$ and $I_{F_{2}}$. The containment $I_{F_{1} \cup F_{2}} \subseteq I_{F_{1}} \cap I_{F_{2}}$ follows immediately from the definitions; we will prove the other containment. To that end, suppose $a \in I_{F_{1}} \cap I_{F_{2}}$. Let $\left\{\phi_{\lambda}^{1}\right\}_{\lambda}$ and $\left\{\phi_{\mu}^{2}\right\}_{\mu}$ be the approximate units for $C_{0}\left(M \backslash F_{1}\right)$ and $C_{0}\left(M \backslash F_{2}\right)$, respectively, that were used in Lemma 2.11, and fix $\epsilon>0$. Then, there exist $\lambda$ and $\mu$ such that $\left\|a-\phi_{\lambda}^{1} \cdot a\right\|<\epsilon$ and $\left\|a-\phi_{\mu}^{2} \cdot a\right\|<\epsilon$; consequently, $\left\|\phi_{\lambda}^{1} \cdot a-\phi_{\mu}^{2} \cdot a\right\|<2 \epsilon$.

Let $\delta=\epsilon /\|a\|$. We will now construct $\phi_{\epsilon} \in C_{0}\left(M \backslash\left(F_{1} \cup F_{2}\right)\right)$ such that $\left\|a-\phi_{\epsilon} \cdot a\right\|<4 \epsilon$, thus showing that $a \in I_{F_{1} \cup F_{2}}$ as claimed.

The open set $U=\left\{m \in M:\left|\phi_{\mu}^{2}(m)\right|<\delta\right\}$ contains $F_{2}$; let $\chi \in C_{0}(M)$ be a bump function that is 1 on $F_{2}$ and 0 off $U$. Then $\phi_{\epsilon}:=\phi_{\lambda}^{1}-\chi \phi_{\lambda}^{1} \in C_{0}\left(M \backslash\left(F_{1} \cup F_{2}\right)\right)$. Moreover,

$$
\begin{aligned}
\left\|a-\left(\phi_{\lambda}^{1}-\chi \phi_{\lambda}^{1}\right) \cdot a\right\| & \leq\left\|a-\left(\phi_{\lambda}^{1}-\chi \phi_{\lambda}^{1}\right) \cdot a-\left(\chi \phi_{\mu}^{2}\right) \cdot a\right\|+\left\|\left(\chi \phi_{\mu}^{2}\right) \cdot a\right\| \\
& \leq\left\|a-\phi_{\lambda}^{1} \cdot a\right\|+\left\|\chi \cdot\left(\phi_{\lambda}^{1} \cdot a-\phi_{\mu}^{2} \cdot a\right)\right\|+\left\|\left(\chi \phi_{\mu}^{2}\right) \cdot a\right\| \\
& <\epsilon+2 \epsilon+\delta\|a\| \\
& =4 \epsilon,
\end{aligned}
$$

since $\chi \phi_{\mu}^{2}$ is only nonzero on $U \backslash F_{2}$, where its maximum modulus is at most $\delta=\epsilon /\|a\|$. Since $\epsilon>0$ is arbitrary, $I_{F_{1}} \cap I_{F_{2}}=I_{F_{1} \cup F_{2}}$ as claimed.

Thus, the map $\phi: A_{F_{1} \cup F_{2}} \rightarrow A_{F_{1}} \oplus A_{F_{2}}$, given by

$$
\phi\left([a]_{F_{1} \cup F_{2}}\right)=[a]_{F_{1}} \oplus[a]_{F_{2}},
$$

is a well-defined, injective $*$-homomorphism. Similarly, the map $\psi$ : $A_{F_{1}} \oplus A_{F_{2}} \rightarrow A_{F_{1} \cap F_{2}}$, given by

$$
\psi\left([a]_{F_{1}} \oplus[b]_{F_{2}}\right)=[a-b]_{F_{1} \cap F_{2}},
$$

is well-defined and onto, since $I_{F_{i}} \subseteq I_{F_{1} \cap F_{2}}$ for $i=1,2$. Since $\operatorname{Im} \phi \subseteq \operatorname{ker} \psi$, by definition, in order to see that the sequence (2.6) is exact, we merely need to check that $\operatorname{Im} \phi \supseteq \operatorname{ker} \psi$. The proof of this inclusion relies on Lemma 2.11.

Suppose $[c]_{F_{1}} \oplus[d]_{F_{2}} \in \operatorname{ker} \psi$. Then $c-d \in I_{F_{1} \cap F_{2}}$, so by Lemma 2.11, there exist $g \in I_{F_{1}}, h \in I_{F_{2}}$ such that $(c-d)-g-h \in I_{F_{1} \cup F_{2}}$. In other 
words,

$$
[c-g]_{F_{1} \cup F_{2}}=[h+d]_{F_{1} \cup F_{2}}=:[b]_{F_{1} \cup F_{2}} .
$$

Since $g \in I_{F_{1}}, h \in I_{F_{2}}$, we know $[c-g]_{F_{1}}=[c]_{F_{1}}$ and $[h+d]_{F_{2}}=[d]_{F_{2}}$. To sum up, if $\psi\left([c]_{F_{1}} \oplus[d]_{F_{2}}\right)=0$, then

$$
[c]_{F_{1}} \oplus[d]_{F_{2}}=[c-g]_{F_{1}} \oplus[d+h]_{F_{2}}=[b]_{F_{1}} \oplus[b]_{F_{2}}=\phi\left([b]_{F_{1} \cup F_{2}}\right),
$$

and

$$
\operatorname{ker} \psi \subseteq \operatorname{Im} \phi
$$

as claimed. In other words, sequence (2.6) is exact.

3. Mayer-Vietoris. In this section, we will translate the results about $C_{0}(M)$-algebras obtained in the previous section into statements about the $K$-theory groups $K_{*}\left(C^{*}(\mathcal{G}, \omega)\right)$ of the $C^{*}$-algebras of twisted group bundles.

$K$-theory (cf., $[\mathbf{1}, \mathbf{1 2}, \mathbf{1 6}]$ ) is a covariant, $\mathbb{Z}_{2}$-graded homotopyinvariant functor from the category of $C^{*}$-algebras to the category of abelian groups. In plain language, this means that $K$-theory associates to each $C^{*}$-algebra $A$ a pair of abelian groups, $K_{0}(A)$ and $K_{1}(A)$. The $K$-theory groups are constructed from equivalence classes of projections in certain $C^{*}$-algebras associated to $A$, and *-homomorphisms $A \rightarrow B$ of $C^{*}$-algebras induce homomorphisms $K_{*}(A) \rightarrow K_{*}(B)$ in such a way that homotopic $*$-homomorphisms induce the same map on $K$-theory.

Among the many useful properties of $K$-theory is the so-called continuity of $K$-theory (cf., [16, Proposition 6.2.9]), which implies that

$$
K_{*}\left(\oplus_{n \in \mathbb{N}} A_{n}\right)=\oplus_{n \in \mathbb{N}} K_{*}\left(A_{n}\right) .
$$

Also relevant to our discussion in this article is the six-term exact sequence in $K$-theory (cf., [1, Theorem 9.3.1], [12, Theorem 12.1.2]): any short exact sequence of $C^{*}$-algebras

$$
0 \longrightarrow J \longrightarrow A \longrightarrow B \longrightarrow 0
$$


induces a six-term exact sequence of the $K$-groups

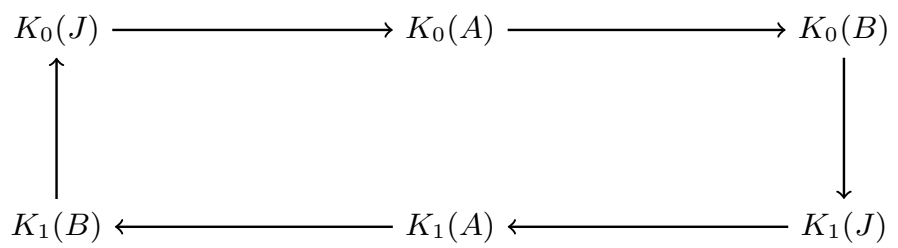

Thus, the short exact sequence (2.6) gives rise to the following six-term exact sequence in $K$-theory:

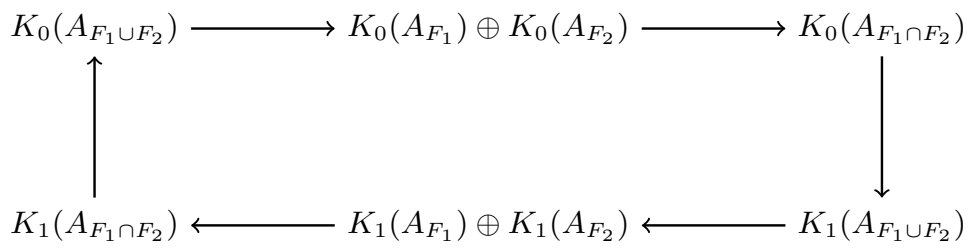

Since $C^{*}(\mathcal{G}, \omega)$ is a $C_{0}(M)$-algebra whenever $\pi: \mathcal{G} \rightarrow M$ is a group bundle, we propose to use this diagram to study the $K$-theory groups associated to a homotopy of 2-cocycles on $\mathcal{G}$. The following definition is a special case of [5, Definition 2.11].

Given a group bundle $\pi: \mathcal{G} \rightarrow M$, we can construct the associated group bundle $\tilde{\pi}: \mathcal{G} \times[0,1] \rightarrow M \times[0,1]$, which has total space $\mathcal{G} \times[0,1]$, and fiber $\pi^{-1}(m)$ over $(m, t) \in M \times[0,1]$ for any $t \in[0,1]$.

Definition 3.1. A homotopy of 2-cocycles on a group bundle $\pi: \mathcal{G} \rightarrow$ $M$ is a 2 -cocycle $\Omega$ on the group bundle $\tilde{\pi}: \mathcal{G} \times[0,1] \rightarrow M \times[0,1]$.

Observe that a homotopy $\Omega$ of 2-cocycles gives rise to a family $\left\{\omega_{t}\right\}_{t \in[0,1]}$ of 2 -cocycles on the original group bundle $\mathcal{G} \rightarrow M$, which varies continuously in $t$ due to the continuity of $\Omega$.

Example 3.2. Suppose that $\pi: V \rightarrow M$ is a vector bundle and that $\sigma: V^{(2)} \rightarrow \mathbb{R}$ is a 2 -form on $V$. The function $\Omega: V^{(2)} \times[0,1] \rightarrow \mathbb{T}$, given by

$$
\Omega((v, w, t))=\exp ^{2 \pi i t \sigma(v, w)},
$$


is a homotopy of 2-cocycles on $V$, with

$$
\omega_{0}(v, w)=1 ; \quad \omega_{1}(v, w)=\exp ^{2 \pi i \sigma(v, w)} .
$$

If $\Omega=\left\{\omega_{t}\right\}_{t \in[0,1]}$ is a homotopy of 2-cocycles on $\mathcal{G}$, we have a natural *-homomorphism

$$
Q_{t}: C^{*}(\mathcal{G} \times[0,1], \Omega) \longrightarrow C^{*}\left(\mathcal{G}, \omega_{t}\right),
$$

for any $t \in[0,1]$, which is given on the dense subalgebra $C_{c}(\mathcal{G} \times[0,1])$ by evaluation at $t$. Observe that, if $F \subseteq M$ is closed, then

$$
Q_{t} \circ q_{F \times[0,1]}=q_{F} \circ Q_{t},
$$

since this equality evidently holds on the dense subalgebra $C_{c}(\mathcal{G} \times[0,1])$, and hence holds in general. Consequently, the diagrams (3.2) for the algebras

$$
A=C^{*}(\mathcal{G} \times[0,1], \Omega), A^{t}=C^{*}\left(\mathcal{G}, \omega_{t}\right)
$$

can be connected into a larger commutative diagram:

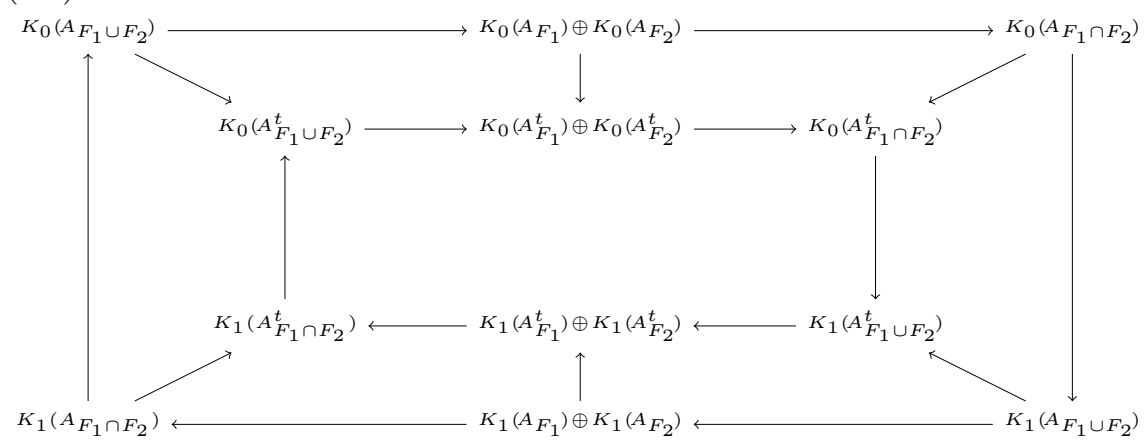

where all of the arrows connecting the inner and outer diagrams arise from the map $Q_{t}$.

Theorem 3.3. Let $\mathcal{G} \rightarrow M$ be a second countable, locally trivial, amenable group bundle, with $\Omega=\left\{\omega_{t}\right\}_{t \in[0,1]}$ a homotopy of 2-cocycles on $\mathcal{G}$. Then

$$
K_{*}\left(C^{*}\left(\mathcal{G}, \omega_{t}\right)\right) \cong K_{*}\left(C^{*}(\mathcal{G} \times[0,1], \Omega)\right)
$$

for any $t \in[0,1]$. 
Proof. We begin by considering the case when $M$ is compact. For each $m \in M$, let $V_{m}$ be a compact neighborhood of $m$ such that $\mathcal{G}$ trivializes over $V_{m}$. Then $\mathcal{G}$ also trivializes over $V_{m} \cap V_{n}$. In other words, $\left.\mathcal{G}\right|_{V_{m}}$ and $\left.\mathcal{G}\right|_{V_{m} \cap V_{n}}$ are transformation groups over compact spaces (with the trivial action of the group $\mathcal{G}_{m} \cong G$ on the spaces $\left.V_{m}, V_{n}, V_{m} \cap V_{n} \subseteq M\right)$. By hypothesis, $\left.\mathcal{G}\right|_{V_{m}}$ is a bundle of amenable groups, and so [14, Theorem 3.3] tells us that $\left.\mathcal{G}\right|_{V_{m}}$ is an amenable groupoid; in other words,

$$
A_{V_{m}}=C^{*}\left(\left.\mathcal{G}\right|_{V_{m}} \times[0,1], \Omega\right) \cong C_{r}^{*}\left(\left.\mathcal{G}\right|_{V_{m}} \times[0,1], \Omega\right) .
$$

According to [5, Theorem 5.1], a homotopy $\Omega=\left\{\omega_{t}\right\}_{t \in[0,1]}$ of 2cocycles on a second countable locally compact transformation group $G \ltimes X$ induces an isomorphism

$$
\left(Q_{t}\right)_{*}: K_{*}\left(C_{r}^{*}(G \ltimes X \times[0,1], \Omega)\right) \rightarrow K_{*}\left(C_{r}^{*}\left(G \ltimes X, \omega_{t}\right)\right),
$$

for any $t \in[0,1]$, as long as $G$ satisfies the Baum-Connes conjecture with coefficients. Applying this result to the cases $G=\mathcal{G}_{m}$ and $X=V_{m}$, since amenable groups satisfy the Baum-Connes conjecture with coefficients, we see that $Q_{t}: A_{V_{m}} \rightarrow A_{V_{m}}^{t}$ induces an isomorphism on $K$-theory, and that the same is true for $Q_{t}: A_{V_{n}} \rightarrow A_{V_{n}}^{t}$ and $Q_{t}: A_{V_{m} \cap V_{n}} \rightarrow A_{V_{m} \cap V_{n}}^{t}$. The Five lemma now implies that $Q_{t}$ : $A_{V_{m} \cup V_{n}} \rightarrow A_{V_{m} \cup V_{n}}^{t}$ induces a $K$-theoretic equivalence.

Iterating this procedure, since the compactness of $M$ implies the existence of a finite cover of $M$ consisting of the sets $V_{m}$, we see that $Q_{t}$ induces an isomorphism

$$
\left(Q_{t}\right)_{*}: K_{*}\left(C^{*}(\mathcal{G} \times[0,1], \Omega)\right) \longrightarrow K_{*}\left(C^{*}\left(\mathcal{G}, \omega_{t}\right)\right),
$$

as claimed.

For the general case, when $M$ is not compact, write

$$
M=\bigcup_{i=1}^{\infty} U_{i}
$$


where $U_{i} \subseteq U_{i+1}$ and $\overline{U_{i}}$ is compact for all $i$. Then $M=F_{1} \cup F_{2}$, where

$$
\begin{aligned}
& F_{1}=\bigcup_{i=0}^{\infty} \overline{U_{2 i+1}} \backslash U_{2 i} ; \\
& F_{2}=\bigcup_{i=1}^{\infty} \overline{U_{2 i}} \backslash U_{2 i-1} .
\end{aligned}
$$

Without loss of generality, we may assume that $\partial U_{i} \cap \partial U_{i+1}=\emptyset$ for all $i$; then, $F_{1}, F_{2}$ and

$$
F_{1} \bigcap F_{2}=\bigcup_{i=1}^{\infty} \partial U_{i}
$$

are each closed sets, consisting of countably many disjoint compact sets.

Consequently, for $F=F_{1}, F_{2}, F_{1} \cap F_{2}$, we see that $C^{*}\left(\left.\mathcal{G}\right|_{F} \times[0,1], \Omega\right)$ and $C^{*}\left(\left.\mathcal{G}\right|_{F}, \omega_{t}\right)$ both break up as a countable direct sum

$$
\begin{aligned}
C^{*}\left(\left.\mathcal{G}\right|_{F} \times[0,1], \Omega\right) & \cong \bigoplus_{n=0}^{\infty} C^{*}\left(\left.\mathcal{G}\right|_{F^{n}} \times[0,1], \Omega\right) ; \\
C^{*}\left(\left.\mathcal{G}\right|_{F}, \omega_{t}\right) & \cong \bigoplus_{n=0}^{\infty} C^{*}\left(\left.\mathcal{G}\right|_{F^{n}}, \omega_{t}\right)
\end{aligned}
$$

where $F^{n}$ is compact for all $n$. Since we established above that, for a compact set $F^{n}$,

$$
\left(Q_{t}\right)_{*}: K_{*}\left(C^{*}\left(\left.\mathcal{G}\right|_{F^{n}} \times[0,1], \Omega\right)\right) \longrightarrow K_{*}\left(C^{*}\left(\left.\mathcal{G}\right|_{F^{n}}, \omega_{t}\right)\right)
$$

is an isomorphism for all $t \in[0,1]$, it follows from equation (3.1) that

$$
\left(Q_{t}\right)_{*}: K_{*}\left(C^{*}\left(\left.\mathcal{G}\right|_{F} \times[0,1], \Omega\right)\right) \longrightarrow K_{*}\left(C^{*}\left(\left.\mathcal{G}\right|_{F}, \omega_{t}\right)\right)
$$

is also an isomorphism for $F=F_{1}, F_{2}, F_{1} \cap F_{2}$. Since $M=F_{1} \cup F_{2}$, the short exact sequence of equation (2.6) combines with the Five lemma (following the same argument given above in the case $M$ is compact) to tell us that

$$
\left(Q_{t}\right)_{*}: K_{*}\left(C^{*}(\mathcal{G} \times[0,1], \Omega)\right) \rightarrow K_{*}\left(C^{*}\left(\mathcal{G}, \omega_{t}\right)\right)
$$

is also an isomorphism. This finishes the proof of Theorem 3.3.

Our main result now follows immediately. 
Corollary 3.4. Any homotopy $\Omega=\left\{\omega_{t}\right\}_{t \in[0,1]}$ of 2-cocycles on a second countable, locally trivial amenable group bundle $\mathcal{G} \rightarrow M$ induces an isomorphism

$$
K_{*}\left(C^{*}\left(\mathcal{G}, \omega_{0}\right)\right) \cong K_{*}\left(C^{*}\left(\mathcal{G}, \omega_{1}\right)\right) .
$$

When we consider the particular case when $V \rightarrow M$ is a vector bundle, we obtain the following generalization of [11, Theorem 1].

Corollary 3.5. Let $V \rightarrow M$ be a vector bundle, and let $\sigma: V^{(2)} \rightarrow \mathbb{R}$ be a bilinear 2-form on $V$. Setting $\omega(v, w)=e^{2 \pi i \sigma(v, w)}$, we have

$$
K_{*}\left(C^{*}(V, \omega)\right) \cong K_{*}\left(C^{*}(V)\right)=K_{*}\left(C_{0}\left(V^{*}\right)\right) .
$$

In particular, if $V$ is even-dimensional, then

$$
K_{*}\left(C^{*}(V, \omega)\right) \cong K_{*}\left(C_{0}(M)\right) .
$$

Acknowledgments. The author would like to thank Nigel Higson for helpful suggestions. I am indebted to him for suggesting this argument for the general case, when $M$ is not compact, in the proof of Theorem 3.3. I am also indebted to my advisor, Erik van Erp, for his support during the writing of this article and throughout my graduate career.

\section{REFERENCES}

1. B. Blackadar, K-theory for operator algebras, Cambridge University Press, Cambridge, 1998.

2. J.H. Brown and A. an Huef, Decomposing the $C^{*}$-algebras of groupoid extensions, Proc. Amer. Math. Soc. 142 (2014), 1261-1274.

3. L.O. Clark and A. an Huef, The representation theory of $C^{*}$-algebras associated to groupoids, Math. Proc. Cambr. Philos. Soc. 153 (2012), 167-191.

4. E. Gillaspy, K-theory and homotopies of 2-cocycles on higher-rank graphs, Pacific J. Math. 278 (2015), 407-426.

5. , K-theory and homotopies of 2-cocycles on transformation groups, J. Oper. Theor. 73 (2015), 465-490.

6. G. Goehle, The Mackey machine for crossed products by regular groupoids, II, Rocky Mountain J. Math. 42 (2012), 873-900.

7. A. an Huef, A. Kumjian and A. Sims, A Dixmier-Douady theorem for Fell algebras, J. Funct. Anal. 260 (2011), 1543-1581.

8. A. Kumjian, On $C^{*}$-diagonals, Canad. J. Math. 38 (1986), 969-1008. 
9. P.S. Muhly, J.N. Renault and D.P. Williams, Continuous-trace groupoid $C^{*}$ algebras, III, Trans. Amer. Math. Soc. 348 (1996), 3621-3641.

10. P.S. Muhly and D.P. Williams, Continuous trace groupoid $C^{*}$-algebras, II, Math. Scandinavia 70 (1992), 127-145.

11. R.J. Plymen, The Weyl bundle, J. Funct. Anal. 49 (1982), 186-197.

12. M. Rørdam, F. Larsen, and N.J. Laustsen, An introduction to $K$-theory for $C^{*}$-algebras, Lond. Math. Soc. 49, Cambridge University Press, Cambridge, 2000.

13. J. Renault, A groupoid approach to $C^{*}$-algebras, Lect. Notes Math. 793, Springer-Verlag, New York, 1980.

14. Topological amenability is a Borel property, Math. Scandinavia $\mathbf{1 1 7}$ (2015), 5-30.

15. J.-L. Tu, P. Xu and C. Laurent-Gengoux, Twisted K-theory of differentiable stacks, Ann. Sci. Ecole Norm. Sup. 37 (2004), 841-910.

16. N.E. Wegge-Olsen, $K$-theory and $C^{*}$-algebras: A friendly approach, Oxford University Press, Oxford, 1993.

17. D.P. Williams, Crossed products of $C^{*}$-algebras, Math. Surv. Mono. 134, American Mathematical Society, Providence, RI, 2007.

Mathematisches Institut Der WWU MÜnster, Einsteinstrasse 62, 48149 Münster, Germany

Email address: gillaspy@uni-muenster.de 